\title{
Logical entropy of dynamical systems
}

\author{
Dagmar Markechová ${ }^{*}$, Abolfazl Ebrahimzadeh² and Zahra Eslami Giski ${ }^{3}$
}

"Correspondence:

dmarkechova@ukf.sk

'Department of Mathematics,

Faculty of Natural Sciences,

Constantine the Philosopher University in Nitra, Nitra, Slovakia

Full list of author information is

available at the end of the article

\begin{abstract}
The main purpose of the paper is to extend the results of Ellerman (Int. J. Semant. Comput. 7:121-145, 2013) to the case of dynamical systems. We define the logical entropy and conditional logical entropy of finite measurable partitions and derive the basic properties of these measures. Subsequently, the suggested concept of logical entropy of finite measurable partitions is used to define the logical entropy of a dynamical system. It is proved that two metrically isomorphic dynamical systems have the same logical entropy. Finally, we provide a logical version of the Kolmogorov-Sinai theorem on generators. So it is shown that by replacing the Shannon entropy function by the logical entropy function we obtain the results analogous to the case of classical Kolmogorov-Sinai entropy theory of dynamical systems,
\end{abstract}

MSC: 28AXX; 37A35; 54C70

Keywords: Measurable partition; Logical entropy; Conditional logical entropy; Measure-preserving transformation; Dynamical system

\section{Introduction}

The concept of entropy plays the central role in information theory [2]; the entropy quantifies the amount of information involved in the value of the outcome of a random process. The entropy has found also applications in other areas, including physics, computer science, statistics, chemistry, biology, sociology, general systems theory and many others and, in addition, the whole new technology and telecommunications industry is based on this quantification of information. The study of the concept of entropy is therefore very important in modern scientific disciplines. As is well known the standard approach in classical information theory is based on the Shannon entropy [3]. We recall that the Shannon entropy of a probability distribution $P=\left\{p_{1}, p_{2}, \ldots, p_{n}\right\}$ is the number $H_{S}(P)=\sum_{i=1}^{n} S\left(p_{i}\right)$, where $S:[0,1] \rightarrow[0, \infty)$ is the Shannon entropy function defined by

$$
S(x)= \begin{cases}-x \log x, & \text { if } x \in(0,1] \\ 0, & \text { if } x=0\end{cases}
$$

The Kolmogorov-Sinai entropy [4-7] provides an important generalization of Shannon entropy; it has strongly influenced understanding of the complexity of dynamical systems. The concept has shown its strength through the highly adequate answers to central problems in the classification of dynamical systems. Two metrically isomorphic dynamical sys-

(c) The Author(s) 2018. This article is distributed under the terms of the Creative Commons Attribution 4.0 International License (http://creativecommons.org/licenses/by/4.0/), which permits unrestricted use, distribution, and reproduction in any medium, provided you give appropriate credit to the original author(s) and the source, provide a link to the Creative Commons license, and indicate if changes were made. 
tems have the same Kolmogorov-Sinai entropy, so the Kolmogorov-Sinai entropy is a tool for distinguishing non-isomorphic dynamical systems.

To address some specific problems, it is preferable to use instead of Shannon entropy an approach based on the concept of logical entropy [1, 8, 9] (see also [10-16]). In [1], the classical logical entropy was discussed by Elllerman as an alternative measure of information. If $P=\left\{p_{1}, p_{2}, \ldots, p_{n}\right\}$ is a probability distribution, then the logical entropy of $P$ is defined by the formula $H_{L}(P)=\sum_{i=1}^{n} L\left(p_{i}\right)$, where $L:[0,1] \rightarrow[0, \infty)$ is the logical entropy function defined, for every $x \in[0,1]$, by the formula:

$$
L(x)=x(1-x) .
$$

The main aim of this paper is to extend the study of logical entropy presented in [1] to the case of dynamical systems; by replacing the Shannon entropy function (1.1) by the logical entropy function (1.2) we construct an isomorphism theory of the Kolmogorov-Sinai type. The paper is organized as follows. Section 2 provides basic definitions and notations, which will be used in the following sections. Our results are presented in Sects. 3 and 4. In Sect. 3, we define and study the logical entropy and conditional logical entropy of finite measurable partitions. In Sect. 4, using the concept of logical entropy of measurable partitions, the notion of logical entropy of a dynamical system is introduced. It is proved that metrically isomorphic dynamical systems have the same logical entropy. Finally, a version of the Kolmogorov-Sinai theorem for the case of the logical entropy is proved. Some concluding remarks are presented in the last section.

\section{Preliminaries}

Modern probability theory is almost exclusively based on the axioms of Kolmogorov [17]. Let us start by recalling Kolmogorov's concept of probability space. We consider a nonempty set $\Omega$, some subsets of $\Omega$ will be called events. Denote by $S$ the family of all events. In the classical probability theory [18] there is assumed that $S$ is a $\sigma$-algebra, i.e., $S$ is a family of subsets of $\Omega$ such that (i) $\Omega \in S$; (ii) if $A \in S$, then $\Omega-A \in S$; (iii) if $A_{n} \in S(n=1,2, \ldots)$, then $\bigcup_{n=1}^{\infty} A_{n} \in S$. The couple $(\Omega, S)$ is said to be a measurable space, the elements of $S$ are said to be measurable.

Let $(\Omega, S)$ be a measurable space. A mapping $\mu: S \rightarrow[0,1]$ is called a probability measure if the following properties are satisfied: (i) $\mu(\Omega)=1$; (ii) $\mu(A) \geq 0$, for every $A \in S$; (iii) if $\left\{A_{n}\right\}_{n=1}^{\infty} \subset S$ such that $A_{i} \cap A_{j}=\emptyset$ whenever $i \neq j$, then $\mu\left(\bigcup_{n=1}^{\infty} A_{n}\right)=\sum_{n=1}^{\infty} \mu\left(A_{n}\right)$. The above described triplet $(\Omega, S, \mu)$ is said to be a probability space.

Further we present definitions of basic terms that we will need in the following sections.

Definition 2.1 ([19]) Let $(\Omega, S)$ be a measurable space. Each finite sequence $\left\{A_{1}, A_{2}, \ldots\right.$, $\left.A_{n}\right\}$ of pairwise disjoint measurable subsets of $\Omega$ such that $\bigcup_{i=1}^{n} A_{i}=\Omega$ is called a (measurable) partition of $\Omega$.

Definition 2.2 ([19]) Let $\alpha=\left\{A_{1}, A_{2}, \ldots, A_{n}\right\}$ and $\beta=\left\{B_{1}, B_{2}, \ldots, B_{m}\right\}$ be two partitions of $\Omega$. The partition $\beta$ is said to be a refinement of $\alpha$ if for each $A_{i} \in \alpha$ there exists a subset $I_{i} \subset\{1,2, \ldots, m\}$ such that $A_{i}=\bigcup_{j \in I_{i}} B_{j}, I_{i} \cap I_{j}=\emptyset$ for $i \neq j$, and $\bigcup_{i=1}^{n} I_{i}=\{1,2, \ldots, m\}$. In this case we write $\alpha \prec \beta$. 
Definition 2.3 ([19]) Given two partitions $\alpha=\left\{A_{1}, A_{2}, \ldots, A_{n}\right\}$ and $\beta=\left\{B_{1}, B_{2}, \ldots, B_{m}\right\}$ of $\Omega$, their join $\alpha \vee \beta$ is defined as the system

$$
\alpha \vee \beta=\left\{A_{i} \cap B_{j} ; i=1,2, \ldots, n, j=1,2, \ldots, m\right\} .
$$

Remark 2.1 It is easy to see that if $\alpha=\left\{A_{1}, A_{2}, \ldots, A_{n}\right\}$ and $\beta=\left\{B_{1}, B_{2}, \ldots, B_{m}\right\}$ are two partitions of $\Omega$, then $\alpha \vee \beta$ is also a partition of $\Omega$. Moreover, $\alpha \prec \alpha \vee \beta$, and $\beta \prec$ $\alpha \vee \beta$. Since the system $\alpha \vee \beta$ is indexed by $\{(i, j) ; i=1,2, \ldots, n, j=1,2, \ldots, m\}$, we put $I_{i}=\{(i, 1), \ldots,(i, m)\}, i=1,2, \ldots, n$. By the assumption we have $\bigcup_{j=1}^{m} B_{j}=\Omega$, therefore we get

$$
A_{i}=\Omega \cap A_{i}=\left(\bigcup_{j=1}^{m} B_{j}\right) \cap A_{i}=\bigcup_{j=1}^{m}\left(B_{j} \cap A_{i}\right)=\bigcup_{(r, j) \in I_{i}}\left(A_{r} \cap B_{j}\right)
$$

for $i=1,2, \ldots, n$. But this means that $\alpha \prec \alpha \vee \beta$.

Definition 2.4 ([19]) Let $\alpha$ and $\beta$ be two partitions of $\Omega$. Then $\alpha \subset^{\circ} \beta$ if for each $A \in \alpha$ there exists $B \in \beta$ such that $\mu(A \Delta B)=0$, where $A \Delta B=(A-B) \cup(B-A)$ denotes the symmetric difference of sets $A, B \in S$. We write $\alpha \approx \beta$ if $\alpha \subset^{\circ} \beta$ and $\beta \subset^{\circ} \alpha$.

Remark 2.2 The relation $\approx$ is an equivalence relation in the family of all measurable partitions of $\Omega$.

\section{Logical entropy of measurable partitions}

In this section, we introduce the concept of logical entropy of measurable partitions and present basic properties of this measure of information. It is shown that it has properties analogous to properties of Shannon's entropy of measurable partitions.

Definition 3.1 Let $\alpha=\left\{A_{1}, A_{2}, \ldots, A_{n}\right\}$ be a partition of $\Omega$. The logical entropy of $\alpha$ is defined as the number

$$
H_{L}(\alpha)=\sum_{i=1}^{n} \mu\left(A_{i}\right)\left(1-\mu\left(A_{i}\right)\right)
$$

Remark 3.1 Since $\sum_{i=1}^{n} \mu\left(A_{i}\right)=\mu\left(\bigcup_{i=1}^{n} A_{i}\right)=\mu(\Omega)=1$, we can also write

$$
H_{L}(\alpha)=1-\sum_{i=1}^{n}\left(\mu\left(A_{i}\right)\right)^{2}
$$

Remark 3.2 Evidently, $H_{L}(\alpha) \geq 0$. For the uniform distribution $p_{i}=\mu\left(A_{i}\right)=\frac{1}{n}, i=$ $1,2, \ldots, n$, over $\alpha=\left\{A_{1}, A_{2}, \ldots, A_{n}\right\}$ the logical entropy $H_{L}(\alpha)$ has its maximum value of $1-\frac{1}{n}$. Thus $0 \leq H_{L}(\alpha) \leq 1-\frac{1}{n}$.

Theorem 3.1 Let $\alpha$ and $\beta$ be two partitions of $\Omega$. Then

(i) $\alpha \prec \beta$ implies $H_{L}(\alpha) \leq H_{L}(\beta)$;

(ii) $H_{L}(\alpha \vee \beta) \geq \max \left(H_{L}(\alpha) ; H_{L}(\beta)\right)$. 
Proof Assume that $\alpha=\left\{A_{1}, A_{2}, \ldots, A_{n}\right\}, \beta=\left\{B_{1}, B_{2}, \ldots, B_{m}\right\}, \alpha \prec \beta$. Then for each $A_{i} \in \alpha$ there exists a subset $I_{i} \subset\{1,2, \ldots, m\}$ such that $A_{i}=\bigcup_{j \in I_{i}} B_{j}, I_{i} \cap I_{j}=\emptyset$ for $i \neq j$, and $\bigcup_{i=1}^{n} I_{i}=$ $\{1,2, \ldots, m\}$. Therefore we can write

$$
\begin{aligned}
H_{L}(\alpha) & =\sum_{i=1}^{n} \mu\left(A_{i}\right)\left(1-\mu\left(A_{i}\right)\right)=\sum_{i=1}^{n}\left(\mu\left(A_{i}\right)-\mu\left(A_{i}\right) \mu\left(A_{i}\right)\right) \\
& =\sum_{i=1}^{n}\left(\mu\left(\bigcup_{j \in I_{i}} B_{j}\right)-\mu\left(\bigcup_{j \in I_{i}} B_{j}\right) \mu\left(\bigcup_{j \in I_{i}} B_{j}\right)\right) \\
& =\sum_{i=1}^{n}\left(\sum_{j \in I_{i}} \mu\left(B_{j}\right)-\sum_{j \in I_{i}} \mu\left(B_{j}\right) \sum_{j \in I_{i}} \mu\left(B_{j}\right)\right) .
\end{aligned}
$$

As a consequence of the inequality $(x+y)^{2} \geq x^{2}+y^{2}$ which holds for all nonnegative real numbers $x, y$, we get

$$
\sum_{j \in I_{i}} \mu\left(B_{j}\right) \sum_{j \in I_{i}} \mu\left(B_{j}\right) \geq \sum_{j \in I_{i}}\left(\mu\left(B_{j}\right)\right)^{2}
$$

for $i=1,2, \ldots, n$. Hence

$$
\begin{aligned}
H_{L}(\alpha) & \leq \sum_{i=1}^{n}\left(\sum_{j \in I_{i}} \mu\left(B_{j}\right)-\sum_{j \in I_{i}}\left(\mu\left(B_{j}\right)\right)^{2}\right) \\
& =\sum_{i=1}^{n} \sum_{j \in I_{i}}\left(\mu\left(B_{j}\right)-\left(\mu\left(B_{j}\right)\right)^{2}\right) \\
& =\sum_{j=1}^{m} \mu\left(B_{j}\right)\left(1-\mu\left(B_{j}\right)\right)=H_{L}(\beta) .
\end{aligned}
$$

Since $\alpha \prec \alpha \vee \beta$ and $\beta \prec \alpha \vee \beta$, the inequality (ii) is a simple consequence of the property (i).

Definition 3.2 If $\alpha=\left\{A_{1}, A_{2}, \ldots, A_{n}\right\}$ and $\beta=\left\{B_{1}, B_{2}, \ldots, B_{m}\right\}$ are two partitions of $\Omega$, then the logical conditional entropy of $\alpha$ assuming a realization of the experiment $\beta$ is defined as the number

$$
H_{L}(\alpha / \beta)=\sum_{i=1}^{n} \sum_{j=1}^{m} \mu\left(A_{i} \cap B_{j}\right)\left(\mu\left(B_{j}\right)-\mu\left(A_{i} \cap B_{j}\right)\right) .
$$

Remark 3.3 The monotonicity of probability measure $\mu$ implies the inequality $\mu\left(A_{i} \cap B_{j}\right) \leq$ $\mu\left(B_{j}\right)$, so it is evident that the logical conditional entropy $H_{L}(\alpha / \beta)$ is a nonnegative number.

Proposition 3.1 Let $\alpha=\left\{A_{1}, A_{2}, \ldots, A_{n}\right\}$ be a partition of $\Omega$. Then, for any $B \in S$, we have $\sum_{i=1}^{n} \mu\left(A_{i} \cap B\right)=\mu(B)$. 
Proof Since $\left(A_{i} \cap B\right) \cap\left(A_{j} \cap B\right)=\emptyset$ whenever $i \neq j$, by the additivity of probability measure $\mu$ we get

$$
\mu(B)=\mu(\Omega \cap B)=\mu\left(\left(\bigcup_{i=1}^{n} A_{i}\right) \cap B\right)=\mu\left(\bigcup_{i=1}^{n}\left(A_{i} \cap B\right)\right)=\sum_{i=1}^{n} \mu\left(A_{i} \cap B\right) .
$$

Remark 3.4 Let $\alpha=\left\{A_{1}, A_{2}, \ldots, A_{n}\right\}$ and $\beta=\left\{B_{1}, B_{2}, \ldots, B_{m}\right\}$ be two partitions of $\Omega$. Since by Proposition 3.1, for $j=1,2, \ldots, m, \sum_{i=1}^{n} \mu\left(A_{i} \cap B_{j}\right)=\mu\left(B_{j}\right)$, we can also write:

$$
H_{L}(\alpha / \beta)=\sum_{j=1}^{m}\left(\mu\left(B_{j}\right)\right)^{2}-\sum_{i=1}^{n} \sum_{j=1}^{m}\left(\mu\left(A_{i} \cap B_{j}\right)\right)^{2} .
$$

Theorem 3.2 Let $\alpha$ and $\beta$ be two partitions of $\Omega$ Then

$$
H_{L}(\alpha / \beta)=H_{L}(\alpha \vee \beta)-H_{L}(\beta) .
$$

Proof Assume that $\alpha=\left\{A_{1}, A_{2}, \ldots, A_{n}\right\}, \beta=\left\{B_{1}, B_{2}, \ldots, B_{m}\right\}$. Let us calculate

$$
\begin{aligned}
H_{L}(\beta)+H_{L}(\alpha / \beta) & =1-\sum_{j=1}^{m}\left(\mu\left(B_{j}\right)\right)^{2}+\sum_{j=1}^{m}\left(\mu\left(B_{j}\right)\right)^{2}-\sum_{i=1}^{n} \sum_{j=1}^{m}\left(\mu\left(A_{i} \cap B_{j}\right)\right)^{2} \\
& =1-\sum_{i=1}^{n} \sum_{j=1}^{m}\left(\mu\left(A_{i} \cap B_{j}\right)\right)^{2}=H_{L}(\alpha \vee \beta) .
\end{aligned}
$$

Remark 3.5 As a simple consequence of the previous theorem we get

$$
H_{L}(\alpha \vee \beta)=H_{L}(\alpha)+H_{L}(\beta / \alpha)
$$

Based on the principle of mathematical induction, we get the following generalization of Eq. (3.2):

$$
H_{L}\left(\alpha_{1} \vee \alpha_{2} \vee \cdots \vee \alpha_{n}\right)=H_{L}\left(\alpha_{1}\right)+\sum_{i=2}^{n} H_{L}\left(\alpha_{i} / \alpha_{1} \vee \cdots \vee \alpha_{i-1}\right)
$$

for every partitions $\alpha_{1}, \alpha_{2}, \ldots, \alpha_{n}$ of $\Omega$. If we put $n=3$, then we have

$$
H_{L}\left(\alpha_{1} \vee \alpha_{2} \vee \alpha_{3}\right)=H_{L}\left(\alpha_{1}\right)+H_{L}\left(\alpha_{2} / \alpha_{1}\right)+H_{L}\left(\alpha_{3} / \alpha_{1} \vee \alpha_{2}\right)
$$

for every partitions $\alpha_{1}, \alpha_{2}, \alpha_{3}$ of $\Omega$.

Theorem 3.3 Let $\alpha$ and $\beta$ be two partitions of $\Omega$. Then

(i) $H_{L}(\alpha / \beta) \leq H_{L}(\alpha)$;

(ii) $H_{L}(\alpha \vee \beta) \leq H_{L}(\alpha)+H_{L}(\beta)$.

Proof Assume that $\alpha=\left\{A_{1}, A_{2}, \ldots, A_{n}\right\}, \beta=\left\{B_{1}, B_{2}, \ldots, B_{m}\right\}$. 
(i) For each $i=1,2, \ldots, n$ we have

$$
\begin{aligned}
& \sum_{j=1}^{m} \mu\left(A_{i} \cap B_{j}\right)\left(\mu\left(B_{j}\right)-\mu\left(A_{i} \cap B_{j}\right)\right) \\
& \leq\left(\sum_{j=1}^{m} \mu\left(A_{i} \cap B_{j}\right)\right)\left(\sum_{j=1}^{m}\left(\mu\left(B_{j}\right)-\mu\left(A_{i} \cap B_{j}\right)\right)\right) \\
& =\mu\left(A_{i}\right)\left(\sum_{j=1}^{m}\left(\mu\left(B_{j}\right)-\mu\left(A_{i} \cap B_{j}\right)\right)\right) \\
& =\mu\left(A_{i}\right)\left(1-\sum_{j=1}^{m} \mu\left(A_{i} \cap B_{j}\right)\right) \\
& =\mu\left(A_{i}\right)\left(1-\mu\left(A_{i}\right)\right) .
\end{aligned}
$$

Therefore we get

$$
\begin{aligned}
H_{L}(\alpha / \beta) & =\sum_{i=1}^{n} \sum_{j=1}^{m} \mu\left(A_{i} \cap B_{j}\right)\left(\mu\left(B_{j}\right)-\mu\left(A_{i} \cap B_{j}\right)\right) \\
& \leq \sum_{i=1}^{n} \mu\left(A_{i}\right)\left(1-\mu\left(A_{i}\right)\right)=H_{L}(\alpha) .
\end{aligned}
$$

(ii) By Eq. (3.2) and the previous part of this theorem, we get

$$
H_{L}(\alpha \vee \beta)=H_{L}(\alpha)+H_{L}(\beta / \alpha) \leq H_{L}(\alpha)+H_{L}(\beta) .
$$

Theorem 3.4 Let $\alpha, \beta$ and $\gamma$ be partitions of $\Omega$. Then

$$
H_{L}(\alpha \vee \beta / \gamma)=H_{L}(\alpha / \gamma)+H_{L}(\beta / \alpha \vee \gamma)
$$

Proof Assume that $\alpha=\left\{A_{1}, A_{2}, \ldots, A_{n}\right\}, \beta=\left\{B_{1}, B_{2}, \ldots, B_{m}\right\}, \gamma=\left\{C_{1}, C_{2}, \ldots, C_{r}\right\}$. Let us calculate

$$
\begin{aligned}
H_{L}(\alpha / \gamma)+H_{L}(\beta / \alpha \vee \gamma)= & \sum_{k=1}^{r}\left(\mu\left(C_{k}\right)\right)^{2}-\sum_{i=1}^{n} \sum_{k=1}^{r}\left(\mu\left(A_{i} \cap C_{k}\right)\right)^{2} \\
& +\sum_{i=1}^{n} \sum_{k=1}^{r}\left(\mu\left(A_{i} \cap C_{k}\right)\right)^{2}-\sum_{j=1}^{m} \sum_{i=1}^{n} \sum_{k=1}^{r}\left(\mu\left(B_{j} \cap A_{i} \cap C_{k}\right)\right)^{2} \\
= & \sum_{k=1}^{r}\left(\mu\left(C_{k}\right)\right)^{2}-\sum_{i=1}^{n} \sum_{j=1}^{m} \sum_{k=1}^{r}\left(\mu\left(A_{i} \cap B_{j} \cap C_{k}\right)\right)^{2} \\
= & H_{L}(\alpha \vee \beta / \gamma) .
\end{aligned}
$$

Using the principle of mathematical induction, we get the following generalization of Theorem 3.4. 
Theorem 3.5 Let $\alpha_{1}, \alpha_{2}, \ldots, \alpha_{n}, \beta$ be partitions of $\Omega$. Then

$$
H_{L}\left(\alpha_{1} \vee \alpha_{2} \vee \cdots \vee \alpha_{n} / \beta\right)=H_{L}\left(\alpha_{1} / \beta\right)+\sum_{i=2}^{n} H_{L}\left(\alpha_{i} / \alpha_{1} \vee \cdots \vee \alpha_{i-1} \vee \beta\right)
$$

Proposition 3.2 Let $\alpha, \beta, \gamma$ be partitions of $\Omega$. Then

(i) $\alpha \prec \beta$ implies $\alpha \vee \gamma \prec \beta \vee \gamma$;

(ii) $\alpha \approx \beta$ implies $\alpha \vee \gamma \approx \beta \vee \gamma$.

Proof Assume that $\alpha=\left\{A_{1}, A_{2}, \ldots, A_{n}\right\}, \beta=\left\{B_{1}, B_{2}, \ldots, B_{m}\right\}, \gamma=\left\{C_{1}, C_{2}, \ldots, C_{r}\right\}$.

(i) Let $\alpha \prec \beta$, i.e., there exists a partition $I_{1}, I_{2}, \ldots, I_{n}$ of the set $\{1,2, \ldots, m\}$ such that $A_{i}=\bigcup_{j \in I_{i}} B_{j}$. A partition $\alpha \vee \gamma=\left\{A_{i} \cap C_{k} ; i=1,2, \ldots, n, k=1,2, \ldots, r\right\}$ is indexed by $\{(i, k)$; $i=1,2, \ldots, n, k=1,2, \ldots, r\}$, hence we put $I_{i, k}=\left\{(j, k) ; j \in I_{i}\right\}$, for $i=1,2, \ldots, n, k=1,2, \ldots, r$. We get

$$
A_{i} \cap C_{k}=\left(\bigcup_{j \in I_{i}} B_{j}\right) \cap C_{k}=\bigcup_{j \in I_{i}}\left(B_{j} \cap C_{k}\right)=\bigcup_{(j, t) \in I_{i, k}}\left(B_{j} \cap C_{t}\right),
$$

for $i=1,2, \ldots, n, k=1,2, \ldots, r$. Therefore $\alpha \vee \gamma \prec \beta \vee \gamma$.

(ii) Let $\alpha \approx \beta$, i.e., $\alpha \subset^{\circ} \beta$ and $\beta \subset^{\circ} \alpha$. From the relation $\alpha \subset^{\circ} \beta$ it follows that for each $A_{i} \in \alpha$ there exists $B_{j} \in \beta$ such that $\mu\left(A_{i}-B_{j}\right)=\mu\left(B_{j}-A_{i}\right)=0$. Hence for each $A_{i} \cap C_{k} \in$ $\alpha \vee \gamma$ there exists $B_{j} \cap C_{k} \in \beta \vee \gamma$ such that

$$
\begin{aligned}
\mu\left(\left(A_{i} \cap C_{k}\right)-\left(B_{j} \cap C_{k}\right)\right) & =\mu\left(A_{i} \cap C_{k} \cap\left(B_{j} \cap C_{k}\right)^{C}\right) \\
& =\mu\left(A_{i} \cap C_{k} \cap\left(B_{j}^{C} \cup C_{k}^{C}\right)\right) \\
& =\mu\left(\left(A_{i} \cap C_{k} \cap B_{j}^{C}\right) \cup\left(A_{i} \cap C_{k} \cap C_{k}^{C}\right)\right) \\
& \leq \mu\left(A_{i} \cap C_{k} \cap B_{j}^{C}\right)+\mu\left(A_{i} \cap C_{k} \cap C_{k}^{C}\right) \\
& \leq \mu\left(A_{i} \cap B_{j}^{C}\right) \\
& =\mu\left(A_{i}-B_{j}\right)=0,
\end{aligned}
$$

and

$$
\begin{aligned}
\mu\left(\left(B_{j} \cap C_{k}\right)-\left(A_{i} \cap C_{k}\right)\right) & =\mu\left(B_{j} \cap C_{k} \cap\left(A_{i} \cap C_{k}\right)^{C}\right) \\
& =\mu\left(B_{j} \cap C_{k} \cap\left(A_{i}^{C} \cup C_{k}^{C}\right)\right) \\
& =\mu\left(\left(B_{j} \cap C_{k} \cap A_{i}^{C}\right) \cup\left(B_{j} \cap C_{k} \cap C_{k}^{C}\right)\right) \\
& \leq \mu\left(B_{j} \cap C_{k} \cap A_{i}^{C}\right)+\mu\left(B_{j} \cap C_{k} \cap C_{k}^{C}\right) \\
& \leq \mu\left(B_{j} \cap A_{i}^{C}\right) \\
& =\mu\left(B_{j}-A_{i}\right)=0 .
\end{aligned}
$$

Hence for each $A_{i} \cap C_{k} \in \alpha \vee \gamma$ there exists $B_{j} \cap C_{k} \in \beta \vee \gamma$ such that

$$
\mu\left(\left(A_{i} \cap C_{k}\right)-\left(B_{j} \cap C_{k}\right)\right)=\mu\left(\left(B_{j} \cap C_{k}\right)-\left(A_{i} \cap C_{k}\right)\right)=0,
$$


i.e.,

$$
\mu\left(\left(A_{i} \cap C_{k}\right) \Delta\left(B_{j} \cap C_{k}\right)\right)=0 .
$$

However, this indicates that $\alpha \vee \gamma \subset^{\circ} \beta \vee \gamma$. Analogously we see that the relation $\beta \subset^{\circ} \alpha$ implies $\beta \vee \gamma \subset^{\circ} \alpha \vee \gamma$. Thus $\alpha \vee \gamma \approx \beta \vee \gamma$.

Theorem 3.6 Let $\alpha, \beta$ and $\gamma$ be partitions of $\Omega$. Then

$$
\alpha \prec \beta \quad \text { implies } \quad H_{L}(\alpha / \gamma) \leq H_{L}(\beta / \gamma) \text {. }
$$

Proof Let $\alpha \prec \beta$. Since by Proposition 3.2 we have $\alpha \vee \gamma \prec \beta \vee \gamma$, according to Theorems 3.2 and 3.1 we get

$$
H_{L}(\alpha / \gamma)=H_{L}(\alpha \vee \gamma)-H_{L}(\gamma) \leq H_{L}(\beta \vee \gamma)-H_{L}(\gamma)=H_{L}(\beta / \gamma) .
$$

Theorem 3.7 Let $\alpha, \beta$ and $\gamma$ be partitions of $\Omega$. Then

(i) $\alpha \subset^{\circ} \beta$ if and only if $H_{L}(\alpha / \beta)=0$;

(ii) $\alpha \approx \beta$ implies $H_{L}(\alpha)=H_{L}(\beta)$;

(iii) $\alpha \approx \beta$ implies $H_{L}(\alpha / \gamma)=H_{L}(\beta / \gamma)$;

(iv) $\alpha \approx \beta$ implies $H_{L}(\gamma / \alpha)=H_{L}(\gamma / \beta)$.

Proof Assume that $\alpha=\left\{A_{1}, A_{2}, \ldots, A_{n}\right\}, \beta=\left\{B_{1}, B_{2}, \ldots, B_{m}\right\}, \gamma=\left\{C_{1}, C_{2}, \ldots, C_{r}\right\}$.

(i) The property is a direct consequence of the equivalence $\alpha \subset^{\circ} \beta \Leftrightarrow \mu\left(A_{i} \cap B_{j}\right)=\mu\left(B_{j}\right)$ or $\mu\left(A_{i} \cap B_{j}\right)=0$, for $i=1,2, \ldots, n$ and $j=1,2, \ldots, m$, proved in [19] (Theorem 4.4).

(ii) By the part (i) the assumption $\alpha \approx \beta$ implies the equalities $H_{L}(\alpha / \beta)=H_{L}(\beta / \alpha)=0$. Therefore, using Eq. (3.2), we get

$$
H_{L}(\alpha \vee \beta)-H_{L}(\beta)=H_{L}(\beta \vee \alpha)-H_{L}(\alpha),
$$

and consequently

$$
H_{L}(\alpha)=H_{L}(\beta) .
$$

(iii) Let $\alpha \approx \beta$. Then, by Proposition $3.2 \alpha \vee \gamma \approx \beta \vee \gamma$, hence by the part (ii) of this theorem we have $H_{L}(\alpha \vee \gamma)=H_{L}(\beta \vee \gamma)$. Hence by Eq. (3.2) we get

$$
H_{L}(\alpha / \gamma)=H_{L}(\alpha \vee \gamma)-H_{L}(\gamma)=H_{L}(\beta \vee \gamma)-H_{L}(\gamma)=H_{L}(\beta / \gamma)
$$

(iv) Let $\alpha \approx \beta$. Then by the part (ii) of this theorem we have $H_{L}(\alpha)=H_{L}(\beta)$. Moreover, by Proposition $3.2 \alpha \vee \gamma \approx \beta \vee \gamma$, hence by the part (ii) of this theorem we get $H_{L}(\alpha \vee \gamma)=$ $H_{L}(\beta \vee \gamma)$. Therefore according to Theorem 3.2 we can write

$$
H_{L}(\gamma / \alpha)=H_{L}(\gamma \vee \alpha)-H_{L}(\alpha)=H_{L}(\beta \vee \gamma)-H_{L}(\beta)=H_{L}(\gamma / \beta)
$$

Note that in the previous theorem (the parts (ii), (iii), (iv)) it is proved that the logical entropy and conditional logical entropy are invariant under the relation $\approx$.

In the following, some illustrative numerical examples are provided. 
Example 3.1 Consider the probability space $(\Omega, S, \mu)$, where $\Omega$ is the unit interval $[0,1]$, $S$ is the $\sigma$-algebra of all Borel subsets of the unit interval $[0,1]$, and the mapping $\mu$ : $S \rightarrow[0,1]$ is the Lebesgue measure, i.e., $\mu([x, y])=y-x$ for any $x, y \in[0,1], x<y$. Evidently, the collections $\alpha=\left\{\left[0, \frac{1}{3}\right),\left[\frac{1}{3}, \frac{2}{3}\right),\left[\frac{2}{3}, 1\right]\right\}$, and $\beta=\left\{\left[0, \frac{1}{4}\right),\left[\frac{1}{4}, \frac{1}{2}\right),\left[\frac{1}{2}, \frac{4}{5}\right),\left[\frac{4}{5}, 1\right]\right\}$ are two measurable partitions of $\Omega$. The join of partitions $\alpha, \beta$ is the collection $\alpha \vee \beta=$ $\left\{\left[0, \frac{1}{4}\right),\left[\frac{1}{4}, \frac{1}{3}\right),\left[\frac{1}{3}, \frac{1}{2}\right),\left[\frac{1}{2}, \frac{2}{3}\right),\left[\frac{2}{3}, \frac{4}{5}\right),\left[\frac{4}{5}, 1\right]\right\}$. By simple calculations we get the logical entropy of these partitions:

$$
\begin{aligned}
& H_{L}(\alpha)=1-\sum_{i=1}^{3}\left(\mu\left(A_{i}\right)\right)^{2}=1-\left(\left(\frac{1}{3}\right)^{2}+\left(\frac{1}{3}\right)^{2}+\left(\frac{1}{3}\right)^{2}\right)=\frac{2}{3} \\
& H_{L}(\beta)=1-\sum_{i=1}^{4}\left(\mu\left(B_{i}\right)\right)^{2}=1-\left(\left(\frac{1}{4}\right)^{2}+\left(\frac{1}{4}\right)^{2}+\left(\frac{3}{10}\right)^{2}+\left(\frac{2}{10}\right)^{2}\right)=\frac{149}{200} \\
& H_{L}(\alpha \vee \beta)=1-\left(\left(\frac{1}{4}\right)^{2}+\left(\frac{1}{12}\right)^{2}+\left(\frac{1}{6}\right)^{2}+\left(\frac{1}{6}\right)^{2}+\left(\frac{2}{15}\right)^{2}+\left(\frac{1}{5}\right)^{2}\right)=\frac{1471}{1800} .
\end{aligned}
$$

The conditional logical entropy of $\alpha$ assuming $\beta$ is the number:

$$
\begin{aligned}
H_{L}(\alpha / \beta)= & \sum_{j=1}^{4}\left(\mu\left(B_{j}\right)\right)^{2}-\sum_{i=1}^{3} \sum_{j=1}^{4}\left(\mu\left(A_{i} \cap B_{j}\right)\right)^{2} \\
= & \left(\frac{1}{4}\right)^{2}+\left(\frac{1}{4}\right)^{2}+\left(\frac{3}{10}\right)^{2}+\left(\frac{2}{10}\right)^{2} \\
& -\left(\left(\frac{1}{4}\right)^{2}+\left(\frac{1}{12}\right)^{2}+\left(\frac{1}{6}\right)^{2}+\left(\frac{1}{6}\right)^{2}+\left(\frac{2}{15}\right)^{2}+\left(\frac{1}{5}\right)^{2}\right) \\
= & \frac{13}{180} .
\end{aligned}
$$

Now it is possible to verify that the equality $H_{L}(\alpha \vee \beta)=H_{L}(\beta)+H_{L}(\alpha / \beta)$ holds.

Example 3.2 Consider the probability space $(\Omega, S, \mu)$, and the measurable partition $\alpha$ of $\Omega$ from the previous example. If we put $\beta=\left\{A_{i} \cap Q, A_{i} \cap Q^{C}, i=1,2, \ldots, n\right\}$, where $Q$ is the set of all rational numbers in the real line $R^{1}$, and $Q^{C}$ denotes the complement of $Q$, then $\beta$ is a measurable partition of $\Omega$. For $i=1,2, \ldots, n$, we have

$$
\mu\left(A_{i} \cap\left(A_{i} \cap Q^{C}\right)\right)=\mu\left(A_{i} \cap Q^{C}\right),
$$

and

$$
\mu\left(A_{i} \cap\left(A_{i} \cap Q\right)\right)=\mu\left(A_{i} \cap Q\right) .
$$

For every $i \neq j$, we get

$$
\mu\left(A_{i} \cap\left(A_{j} \cap Q^{C}\right)\right)=\mu\left(A_{i} \cap A_{j} \cap Q^{C}\right)=\mu(\emptyset)=0 .
$$


Therefore we conclude that, for every $A \in \alpha, B \in \beta, \mu(A \cap B)=\mu(B)$ or $\mu(A \cap B)=0$. But this means that $\alpha \subset^{\circ} \beta$. Hence we get

$$
\begin{aligned}
H_{L}(\alpha / \beta) & =\sum_{j=1}^{m}\left(\mu\left(B_{j}\right)\right)^{2}-\sum_{i=1}^{n} \sum_{j=1}^{m}\left(\mu\left(A_{i} \cap B_{j}\right)\right)^{2} \\
& =\sum_{j=1}^{m}\left(\mu\left(B_{j}\right)\right)^{2}-\sum_{j=1}^{m}\left(\mu\left(B_{j}\right)\right)^{2}=0 .
\end{aligned}
$$

In the same way we see that $\beta \subset^{\circ} \alpha$, and $H_{L}(\beta / \alpha)=0$. Since $\alpha \subset^{\circ} \beta$ and $\beta \subset^{\circ} \alpha, \alpha \approx \beta$. Evidently, $H_{L}(\alpha)=H_{L}(\beta)$.

Theorem 3.8 If partitions $\alpha=\left\{A_{1}, A_{2}, \ldots, A_{n}\right\}$ and $\beta=\left\{B_{1}, B_{2}, \ldots, B_{m}\right\}$ of $\Omega$ are statistically independent, i.e., $\mu\left(A_{i} \cap B_{j}\right)=\mu\left(A_{i}\right) \cdot \mu\left(B_{j}\right)$, for $i=1,2, \ldots, n$ and $j=1,2, \ldots, m$, then

$$
1-H_{L}(\alpha \vee \beta)=\left(1-H_{L}(\alpha)\right) \cdot\left(1-H_{L}(\beta)\right) \text {. }
$$

Proof Let us calculate

$$
\begin{aligned}
1-H_{L}(\alpha \vee \beta) & =\sum_{i=1}^{n} \sum_{j=1}^{m}\left(\mu\left(A_{i} \cap B_{j}\right)\right)^{2} \\
& =\sum_{i=1}^{n} \sum_{j=1}^{m}\left(\mu\left(A_{i}\right) \cdot \mu\left(B_{j}\right)\right)^{2} \\
& =\sum_{i=1}^{n}\left(\mu\left(A_{i}\right)\right)^{2} \sum_{j=1}^{m}\left(\mu\left(B_{j}\right)\right)^{2} \\
& =\left(1-H_{L}(\alpha)\right) \cdot\left(1-H_{L}(\beta)\right) .
\end{aligned}
$$

Remark 3.6 By contrast, in the case of Shannon's entropy, the additivity applies: for statistically independent partitions $\alpha, \beta$ of $\Omega$

$$
H_{S}(\alpha \vee \beta)=H_{S}(\alpha)+H_{S}(\beta) .
$$

\section{Logical entropy of dynamical systems}

In this section using the concept of logical entropy of measurable partitions we define the logical entropy of dynamical systems. Recall that a dynamical system in the sense of classical probability theory [19] is a quadruple $(\Omega, S, \mu, T)$, where $(\Omega, S, \mu)$ is a probability space and $T: \Omega \rightarrow \Omega$ is a measure preserving transformation (i.e., $E \in S$ implies $T^{-1}(E) \in S$ and $\left.\mu\left(T^{-1}(E)\right)=\mu(E)\right)$. If $\alpha=\left\{A_{1}, A_{2}, \ldots, A_{n}\right\}$ is a partition of $\Omega$, then by $T^{-1}(\alpha)$ the partition $\left\{T^{-1}\left(A_{1}\right), T^{-1}\left(A_{2}\right), \ldots, T^{-1}\left(A_{n}\right)\right\}$ is denoted. By Eq. (3.1) the logical entropy of partition $\bigvee_{i=0}^{n-1} T^{-i}(\alpha)=\alpha \vee T^{-1}(\alpha) \vee \cdots \vee T^{-(n-1)}(\alpha)$ can also be found.

We note that fuzzy generalizations of the notion of a dynamical system have been introduced and studied e.g., in [20-26]. Notice that while in the papers [20-22, 26] the authors deal with the Shannon and Kolmogorov-Sinai entropy of fuzzy dynamical systems, in the paper [23] the logical entropy of fuzzy dynamical systems has been studied. We remark that some of the results of the article [23] are fuzzy generalizations of the results provided in Sects. 3 and 4 of the present paper. 
Proposition 4.1 Let any dynamical system $(\Omega, S, \mu, T)$ be given. If $\alpha, \beta$ are partitions of $\Omega$, then

(i) $T^{-1}(\alpha \vee \beta)=T^{-1}(\alpha) \vee T^{-1}(\beta)$;

(ii) $\alpha \prec \beta$ implies $T^{-1}(\alpha) \prec T^{-1}(\beta)$.

Proof Assume that $\alpha=\left\{A_{1}, A_{2}, \ldots, A_{n}\right\}, \beta=\left\{B_{1}, B_{2}, \ldots, B_{m}\right\}$. The property (i) follows from the equality $T^{-1}\left(A_{i} \cap B_{j}\right)=T^{-1}\left(A_{i}\right) \cap T^{-1}\left(B_{j}\right), i=1,2, \ldots, n, j=1,2, \ldots, m$.

(ii) Assume that $\alpha \prec \beta$. Then for each $A_{i} \in \alpha$ there exists a subset $I_{i} \subset\{1,2, \ldots, m\}$ such that $A_{i}=\bigcup_{j \in I_{i}} B_{j}, I_{i} \cap I_{j}=\emptyset$ for $i \neq j$, and $\bigcup_{i=1}^{n} I_{i}=\{1,2, \ldots, m\}$. Therefore we can write

$$
T^{-1}\left(A_{i}\right)=T^{-1}\left(\bigcup_{j \in I_{i}} B_{j}\right)=\bigcup_{j \in I_{i}} T^{-1}\left(B_{j}\right), \quad i=1,2, \ldots, n .
$$

However, this means that $T^{-1}(\alpha) \prec T^{-1}(\beta)$.

Theorem 4.1 Let any dynamical system $(\Omega, S, \mu, T)$ be given. If $\alpha, \beta$ are partitions of $\Omega$, then the following properties are satisfied:

(i) $H_{L}\left(T^{-r}(\alpha)\right)=H_{L}(\alpha), r=0,1,2, \ldots$;

(ii) $H_{L}\left(T^{-r}(\alpha) / T^{-r}(\beta)\right)=H_{L}(\alpha / \beta), r=0,1,2, \ldots$;

(iii) $H_{L}\left(\bigvee_{i=0}^{n-1} T^{-i}(\alpha)\right)=H_{L}(\alpha)+\sum_{j=1}^{n-1} H_{L}\left(\alpha / \bigvee_{i=1}^{j} T^{-i}(\alpha)\right)$.

Proof (i) Assume that $\alpha=\left\{A_{1}, A_{2}, \ldots, A_{n}\right\}$. Since $\mu\left(T^{-r}\left(A_{i}\right)\right)=\mu\left(A_{i}\right)$, for $i=1,2, \ldots, n$, and $r=0,1,2, \ldots$, we get

$$
H_{L}\left(T^{-r}(\alpha)\right)=1-\sum_{i=1}^{n}\left(\mu\left(T^{-r}\left(A_{i}\right)\right)\right)^{2}=1-\sum_{i=1}^{n}\left(\mu\left(A_{i}\right)\right)^{2}=H_{L}(\alpha) .
$$

(ii) Assume that $\alpha=\left\{A_{1}, A_{2}, \ldots, A_{n}\right\}, \beta=\left\{B_{1}, B_{2}, \ldots, B_{m}\right\}$. Then

$$
\begin{aligned}
H_{L}\left(T^{-r}(\alpha) / T^{-r}(\beta)\right) & =\sum_{j=1}^{m}\left(\mu\left(T^{-r}\left(B_{j}\right)\right)\right)^{2}-\sum_{i=1}^{n} \sum_{j=1}^{m}\left(\mu\left(T^{-r}\left(A_{i} \cap B_{j}\right)\right)\right)^{2} \\
& =\sum_{j=1}^{m}\left(\mu\left(B_{j}\right)\right)^{2}-\sum_{i=1}^{n} \sum_{j=1}^{m}\left(\mu\left(A_{i} \cap B_{j}\right)\right)^{2} \\
& =H_{L}(\alpha / \beta) .
\end{aligned}
$$

(iii) We will prove by mathematical induction. For the case of $n=2$, the equality (iii) is a simple consequence of Eq. (3.2). We assume that the statement holds for a given $n \in \mathrm{N}$ and we prove it is true for $n+1$. By the property (i) we have

$$
H_{L}\left(\bigvee_{i=1}^{n} T^{-i}(\alpha)\right)=H_{L}\left(T^{-1}\left(\bigvee_{i=0}^{n-1} T^{-i}(\alpha)\right)\right)=H_{L}\left(\bigvee_{i=0}^{n-1} T^{-i}(\alpha)\right)
$$


Therefore using Eq. (3.2) and the induction assumption we get

$$
\begin{aligned}
H_{L}\left(\bigvee_{i=0}^{n} T^{-i}(\alpha)\right) & =H_{L}\left(\left(\bigvee_{i=1}^{n} T^{-i}(\alpha)\right) \vee \alpha\right)=H_{L}\left(\bigvee_{i=1}^{n} T^{-i}(\alpha)\right)+H_{L}\left(\alpha / \bigvee_{i=1}^{n} T^{-i}(\alpha)\right) \\
& =H_{L}\left(\bigvee_{i=0}^{n-1} T^{-i}(\alpha)\right)+H_{L}\left(\alpha / \bigvee_{i=1}^{n} T^{-i}(\alpha)\right) \\
& =H_{L}(\alpha)+\sum_{j=1}^{n-1} H_{L}\left(\alpha / \bigvee_{i=1}^{j} T^{-i}(\alpha)\right)+H_{L}\left(\alpha / \bigvee_{i=1}^{n} T^{-i}(\alpha)\right) \\
& =H_{L}(\alpha)+\sum_{j=1}^{n} H_{L}\left(\alpha / \bigvee_{i=1}^{j} T^{-i}(\alpha)\right) .
\end{aligned}
$$

The proof is complete.

In the following, we define the logical entropy of a dynamical system $(\Omega, S, \mu, T)$. We will begin by defining the logical entropy of a measure preserving transformation $T$ relative to a partition $\alpha$. Later we shall remove the dependence on $\alpha$ to obtain the logical entropy of a dynamical system $(\Omega, S, \mu, T)$. We first need the following standard analytic lemma.

Lemma 4.1 ([19], Theorem 4.9) Let $\left\{u_{n}\right\}_{n=1}^{\infty}$ be a sequence of nonnegative real numbers such that $u_{r+s} \leq u_{r}+u_{s}$ for every $r, s \in \mathrm{N}$. Then $\lim _{n \rightarrow \infty} \frac{1}{n} u_{n}$ exists.

Theorem 4.2 Let $(\Omega, S, \mu, T)$ be a dynamical system and $\alpha$ be a measurable partition of $\Omega$. Then $\lim _{n \rightarrow \infty} \frac{1}{n} H_{L}\left(\bigvee_{i=0}^{n-1} T^{-i}(\alpha)\right)$ exists.

Proof Put $u_{n}=H_{L}\left(\bigvee_{i=0}^{n-1} T^{-i}(\alpha)\right)$. Evidently, the sequence $\left\{u_{n}\right\}_{n=1}^{\infty}$ is a sequence of nonnegative real numbers. We prove that $u_{r+s} \leq u_{r}+u_{s}$ for every $r, s \in \mathrm{N}$. According to the subadditivity of logical entropy (Theorem 3.3(ii)), and the property (i) of the previous theorem, for every $r, s \in \mathrm{N}$, we have

$$
\begin{aligned}
u_{r+s} & =H_{L}\left(\bigvee_{i=0}^{r+s-1} T^{-i}(\alpha)\right) \leq H_{L}\left(\bigvee_{i=0}^{r-1} T^{-i}(\alpha)\right)+H_{L}\left(\bigvee_{i=r}^{r+s-1} T^{-i}(\alpha)\right) \\
& =u_{r}+H_{L}\left(\bigvee_{i=0}^{s-1} T^{-(r+i)}(\alpha)\right)=u_{r}+H_{L}\left(T^{-r}\left(\bigvee_{i=0}^{s-1} T^{-i}(\alpha)\right)\right) \\
& =u_{r}+H_{L}\left(\bigvee_{i=0}^{s-1} T^{-i}(\alpha)\right)=u_{r}+u_{s} .
\end{aligned}
$$

Hence by Lemma 4.1, $\lim _{n \rightarrow \infty} \frac{1}{n} H_{L}\left(\bigvee_{i=0}^{n-1} T^{-i}(\alpha)\right)$ exists.

Definition 4.1 Let $(\Omega, S, \mu, T)$ be a dynamical system and $\alpha$ be a measurable partition of $\Omega$. The logical entropy of $T$ with respect to $\alpha$ is defined by

$$
h_{L}(T, \alpha)=\lim _{n \rightarrow \infty} \frac{1}{n} H_{L}\left(\bigvee_{i=0}^{n-1} T^{-i}(\alpha)\right) \text {. }
$$


Remark 4.1 Evidently, $h_{L}(T, \alpha) \geq 0$.

Theorem 4.3 Let $(\Omega, S, \mu, T)$ be a dynamical system and $\alpha$ and $\beta$ be measurable partitions of $\Omega$ such that $\alpha \prec \beta$. Then $h_{L}(T, \alpha) \leq h_{L}(T, \beta)$.

Proof The assumption $\alpha \prec \beta$ implies the relation $\bigvee_{i=0}^{n-1} T^{-i}(\alpha) \prec \bigvee_{i=0}^{n-1} T^{-i}(\beta)$, for every $n \in \mathrm{N}$. According to the property (i) of Theorem 3.1, we get

$$
H_{L}\left(\bigvee_{i=0}^{n-1} T^{-i}(\alpha)\right) \leq H_{L}\left(\bigvee_{i=0}^{n-1} T^{-i}(\beta)\right)
$$

Consequently, dividing by $n$ and letting $n \rightarrow \infty$, we conclude that

$$
h_{L}(T, \alpha) \leq h_{L}(T, \beta) \text {. }
$$

Definition 4.2 The logical entropy of a dynamical system $(\Omega, S, \mu, T)$ is defined by

$$
h_{L}(T)=\sup \left\{h_{L}(T, \alpha) ; \alpha \text { is a partition of } \Omega\right\} \text {. }
$$

Example 4.1 The system $(\Omega, S, \mu, I)$, where $I: \Omega \rightarrow \Omega$ is the identity map, is a trivial case of a dynamical system. The operation $\bigvee$ is idempotent, therefore, for every partition $\alpha$ of $\Omega$, we have

$$
h_{L}(I, \alpha)=\lim _{n \rightarrow \infty} \frac{1}{n} H_{L}\left(\bigvee_{i=0}^{n-1} I^{-i}(\alpha)\right)=\lim _{n \rightarrow \infty} \frac{1}{n} H_{L}(\alpha)=0
$$

Thus the logical entropy of $(\Omega, S, \mu, I)$ is the number

$$
h_{L}(I)=\sup \left\{h_{L}(I, \alpha) ; \alpha \text { is a partition of } \Omega\right\}=0 \text {. }
$$

Theorem 4.4 Let $(\Omega, S, \mu, T)$ be a dynamical system. Then, for any integer $k>0, h_{L}\left(T^{k}\right)=$ $k \cdot h_{L}(T)$.

Proof For each partition $\alpha$ of $\Omega$ we have

$$
\begin{aligned}
h_{L}\left(T^{k}, \bigvee_{i=0}^{k-1} T^{-i}(\alpha)\right) & =\lim _{n \rightarrow \infty} \frac{1}{n} H_{L}\left(\bigvee_{j=0}^{n-1}\left(T^{k}\right)^{-j}\left(\bigvee_{i=0}^{k-1} T^{-i}(\alpha)\right)\right) \\
& =\lim _{n \rightarrow \infty} \frac{1}{n} H_{L}\left(\bigvee_{j=0}^{n-1} \bigvee_{i=0}^{k-1} T^{-(k j+i)}(\alpha)\right)=\lim _{n \rightarrow \infty} \frac{1}{n} H_{L}\left(\bigvee_{i=0}^{n k-1} T^{-i}(\alpha)\right) \\
& =\lim _{n \rightarrow \infty} \frac{n k}{n} \frac{1}{n k} H_{L}\left(\bigvee_{i=0}^{n k-1} T^{-i}(\alpha)\right)=k \cdot h_{L}(T, \alpha) .
\end{aligned}
$$


Therefore we get

$$
\begin{aligned}
k \cdot h_{L}(T) & =k \cdot \sup \left\{h_{L}(T, \alpha) ; \alpha \text { is a partition of } \Omega\right\} \\
& =\sup \left\{h_{L}\left(T^{k}, \bigvee_{i=0}^{k-1} T^{-i}(\alpha)\right) ; \alpha \text { is a partition of } \Omega\right\} \\
& \leq \sup \left\{h_{L}\left(T^{k}, \beta\right) ; \beta \text { is a partition of } \Omega\right\}=h_{L}\left(T^{k}\right) .
\end{aligned}
$$

On the other hand $\alpha \prec \bigvee_{i=0}^{k-1} T^{-i}(\alpha)$, and therefore

$$
h_{L}\left(T^{k}, \alpha\right) \leq h_{L}\left(T^{k}, \bigvee_{i=0}^{k-1} T^{-i}(\alpha)\right)=k \cdot h_{L}(T, \alpha)
$$

It follows that

$$
\begin{aligned}
h_{L}\left(T^{k}\right) & =\sup \left\{h_{L}\left(T^{k}, \alpha\right) ; \alpha \text { is a partition of } \Omega\right\} \\
& \leq k \cdot \sup \left\{h_{L}(T, \alpha) ; \alpha \text { is a partition of } \Omega\right\}=k \cdot h_{L}(T) .
\end{aligned}
$$

Corollary 4.1 Let $(\Omega, S, \mu, T)$ be a dynamical system and let there exist an integer $k>0$ such that $T^{k}$ is the identity map. Then $h_{L}(T)=0$.

Proof Let $k>0$ be an integer such that $T^{k}=I$. Then we have

$$
h_{L}(T)=\frac{1}{k} h_{L}\left(T^{k}\right)=\frac{1}{k} h_{L}(I)=0 .
$$

Theorem 4.5 Let $(\Omega, S, \mu, T)$ be a dynamical system and $\alpha$ be a measurable partition of $\Omega$. Then, for any integer $k>0$,

$$
h_{L}(T, \alpha)=h_{L}\left(T, \bigvee_{i=0}^{k} T^{-i}(\alpha)\right)
$$

Proof Let $\alpha$ be a measurable partition of $\Omega$. Then, for any integer $k>0$, we have

$$
\begin{aligned}
h_{L}\left(T, \bigvee_{i=0}^{k} T^{-i}(\alpha)\right) & =\lim _{n \rightarrow \infty} \frac{1}{n} H_{L}\left(\bigvee_{j=0}^{n-1} T^{-j}\left(\bigvee_{i=0}^{k} T^{-i}(\alpha)\right)\right) \\
& =\lim _{n \rightarrow \infty} \frac{k+n}{n} \cdot \frac{1}{k+n} H_{L}\left(\bigvee_{t=0}^{k+n-1} T^{-t}(\alpha)\right) \\
& =\lim _{n \rightarrow \infty} \frac{1}{k+n} H_{L}\left(\bigvee_{t=0}^{k+n-1} T^{-t}(\alpha)\right)=h_{L}(T, \alpha) .
\end{aligned}
$$

In the following part, we prove that two metrically isomorphic dynamical systems have the same logical entropy. First recall the definition of what it means that two dynamical systems are metrically isomorphic.

Definition 4.3 We say that two dynamical systems $\left(\Omega_{1}, S_{1}, \mu_{1}, T_{1}\right),\left(\Omega_{2}, S_{2}, \mu_{2}, T_{2}\right)$ are metrically isomorphic if there exist $X_{1} \in S_{1}$ and $X_{2} \in S_{2}$ such that 
(i) $T_{1} X_{1} \subset X_{1}, T_{2} X_{2} \subset X_{2}$;

(ii) $\mu_{1}\left(X_{1}\right)=1, \mu_{2}\left(X_{2}\right)=1$,

and there exists a bijective map $\psi: X_{1} \rightarrow X_{2}$ such that

(iii) $\psi, \psi^{-1}$ are measure preserving;

(iv) $\psi \circ T_{1}=T_{2} \circ \psi$.

Theorem 4.6 If dynamical systems $\left(\Omega_{1}, S_{1}, \mu_{1}, T_{1}\right),\left(\Omega_{2}, S_{2}, \mu_{2}, T_{2}\right)$ are metrically isomorphic, then $h_{L}\left(T_{1}\right)=h_{L}\left(T_{2}\right)$.

Proof Let $X_{1} \subset \Omega_{1}, X_{2} \subset \Omega_{2}$ and $\psi: X_{1} \rightarrow X_{2}$ be as in the previous definition. If $\alpha=$ $\left\{A_{1}, A_{2}, \ldots, A_{n}\right\}$ is a measurable partition of $\Omega_{2}$, then (changing it on a set of measure zero if necessary) it is also a measurable partition of $X_{2}$. The inverse image $\psi^{-1} \alpha=\left\{\psi^{-1}\left(A_{i}\right)\right.$; $\left.A_{i} \in \alpha\right\}$ is a measurable partition of $X_{1}$ and hence of $\Omega_{1}$. Moreover,

$$
\begin{aligned}
H_{L}\left(\psi^{-1} \alpha\right) & =\sum_{i=1}^{n} \mu_{1}\left(\psi^{-1}\left(A_{i}\right)\right)\left(1-\mu_{1}\left(\psi^{-1}\left(A_{i}\right)\right)\right) \\
& =\sum_{i=1}^{n} \mu_{2}\left(A_{i}\right)\left(1-\mu_{2}\left(A_{i}\right)\right)=H_{L}(\alpha) .
\end{aligned}
$$

Hence we can write

$$
H_{L}\left(\bigvee_{i=0}^{n-1} T_{1}^{-i}\left(\psi^{-1} \alpha\right)\right)=H_{L}\left(\psi^{-1} \bigvee_{i=0}^{n-1} T_{2}^{-i}(\alpha)\right)=H_{L}\left(\bigvee_{i=0}^{n-1} T_{2}^{-i}(\alpha)\right)
$$

Therefore, dividing by $n$ and letting $n \rightarrow \infty$, we get

$$
h_{L}\left(T_{2}, \alpha\right)=h_{L}\left(T_{1}, \psi^{-1} \alpha\right)
$$

Thus

$$
\left\{h_{L}\left(T_{2}, \alpha\right) ; \alpha \text { is a partition of } \Omega_{2}\right\} \subset\left\{h_{L}\left(T_{1}, \beta\right) ; \beta \text { is a partition of } \Omega_{1}\right\} \text {, }
$$

and consequently

$$
\begin{aligned}
h_{L}\left(T_{2}\right) & =\sup \left\{h_{L}\left(T_{2}, \alpha\right) ; \alpha \text { is a partition of } \Omega_{2}\right\} \\
& \leq \sup \left\{h_{L}\left(T_{1}, \beta\right) ; \beta \text { is a partition of } \Omega_{1}\right\}=h_{L}\left(T_{1}\right) .
\end{aligned}
$$

By symmetry, we also have $h_{L}\left(T_{1}\right) \leq h_{L}\left(T_{2}\right)$. The proof is completed.

Remark 4.2 From Theorem 4.6 it follows that if $h_{L}\left(T_{1}\right) \neq h_{L}\left(T_{2}\right)$, then the corresponding dynamical systems $\left(\Omega_{1}, S_{1}, \mu_{1}, T_{1}\right),\left(\Omega_{2}, S_{2}, \mu_{2}, T_{2}\right)$ are metrically non-isomorphic. This means that the logical entropy distinguishes metrically non-isomorphic dynamical systems; so we have acquired an alternative tool for distinguishing non-isomorphic dynamical systems. This result is illustrated in the following example.

Example 4.2 Consider the probability space $(\Omega, S, \mu)$, where $\Omega$ is the unit interval $[0,1]$, $S$ is the $\sigma$-algebra of all Borel subsets of the unit interval $[0,1]$, and $\mu: S \rightarrow[0,1]$ is the 
Lebesgue measure, i.e., $\mu([x, y])=y-x$ for any $x, y \in[0,1], x<y$. Let $c \in(0,1)$, and the mapping $T_{c}:[0,1] \rightarrow[0,1]$ is defined by the formula $T_{c}(x)=x+c(\bmod 1)$. The logical entropy distinguishes metrically non-isomorphic dynamical systems $\left(\Omega, S, \mu, T_{c}\right)$ for different $c$. Namely, $h_{L}\left(T_{c}\right)=0$, if $c=1 / 2$, but $h_{L}\left(T_{c}\right)>0$ for $c=1-\sqrt{2}$.

The well-known Kolmogorov-Sinai theorem on generators [19] (see also [27, 28]) is the main tool for calculating the entropy of dynamical system. We conclude our contribution with the formulation of this theorem for the case of logical entropy.

Definition 4.4 A partition $\gamma$ of $\Omega$ is said to be a generator of a dynamical system $(\Omega, S, \mu, T)$, if to any partition $\alpha$ of $\Omega$ there exists an integer $k>0$ such that $\alpha \prec \bigvee_{i=0}^{k} T^{-i}(\gamma)$.

Theorem 4.7 Let $\gamma$ be a generator of a dynamical system $(\Omega, S, \mu, T)$. Then

$$
h_{L}(T)=h_{L}(T, \gamma) \text {. }
$$

Proof Let $\gamma$ be a generator of a dynamical system $(\Omega, S, \mu, T)$. Then to any partition $\alpha$ of $\Omega$ there exists an integer $k>0$ such that $\alpha \prec \bigvee_{i=0}^{k} T^{-i}(\gamma)$.

Hence by Theorems 4.3 and 4.5 we get

$$
h_{L}(T, \alpha) \leq h_{L}\left(T, \bigvee_{i=0}^{k} T^{-i}(\gamma)\right)=h_{L}(T, \gamma)
$$

Therefore

$$
h_{L}(T)=\sup \left\{h_{L}(T, \alpha) ; \alpha \text { is a partition of } \Omega\right\}=h_{L}(T, \gamma) .
$$

\section{Conclusions}

In this contribution we have extended the results of Ellerman presented in [1] to the case of dynamical systems. Our results are given in Sects. 3 and 4. In Sect. 3 we introduced the notions of logical entropy and logical conditional entropy of finite measurable partitions of a probability space and we examined basic properties of the proposed measures. We have provided some numerical examples to illustrate the results as well. In Sect. 4, the results of the previous part were used to introduce the concept of logical entropy of the dynamical system. It has been shown that two metrically isomorphic dynamical systems have the same logical entropy. Since the logical entropy distinguishes metrically non-isomorphic dynamical systems, we have acquired an alternative tool for distinguishing non-isomorphic dynamical systems. This result is illustrated by Example 4.2. Finally, we have proved a logical version of the Kolmogorov-Sinai theorem on generators (Theorem 4.7). In this study, it has been shown that by replacing the Shannon entropy function by the logical entropy function we obtain the results analogous to the case of classical Kolmogorov-Sinai entropy theory.

\section{Acknowledgements}

The authors thank the editor and the anonymous referees for their valuable comments and suggestions which have significantly improved the quality and presentation of this paper. 


\section{Competing interests}

The authors declare that they have no competing interests.

\section{Authors' contributions}

All authors contributed equally and significantly in writing this article. All authors read and approved the final manuscript.

\section{Author details}

${ }^{1}$ Department of Mathematics, Faculty of Natural Sciences, Constantine the Philosopher University in Nitra, Nitra, Slovakia.

${ }^{2}$ Department of Mathematics, Zahedan Branch, Islamic Azad University, Zahedan, Iran. ${ }^{3}$ Department of Mathematics,

Sirjan Branch, Islamic Azad University, Sirjan, Iran.

\section{Publisher's Note}

Springer Nature remains neutral with regard to jurisdictional claims in published maps and institutional affiliations.

Received: 12 September 2017 Accepted: 15 February 2018 Published online: 27 February 2018

\section{References}

1. Ellerman, D.: An introduction to logical entropy and its relation to Shannon entropy. Int. J. Semant. Comput. 7, $121-145(2013)$

2. Gray, R.M.: Entropy and Information Theory. Springer, Berlin (2009)

3. Shannon, C.E.: A mathematical theory of communication. Bell Syst. Tech. J. 27, 379-423 (1948)

4. Kolmogorov, A.N.: New metric invariant of transitive dynamical systems and automorphisms of Lebesgue spaces. Dokl. Russ. Acad. Sci. 119, 861-864 (1958)

5. Sinai, Y.G.: Ergodic Theory with Applications to Dynamical Systems and Statistical Mechanics. Springer, Berlin (1990)

6. Sinai, Y.G.: On the notion of the entropy of dynamical systems. Dokl. Russ. Acad. Sci. 124, 768-771 (1959)

7. Sinai, Y.G.: Theory of Dynamical Systems. Part I: Ergodic Theory. Lecture Notes Series, vol. 23. Matematisk Institut, Aarhus Universitet, Aarhus (1970)

8. Ellerman, D.: Counting distinctions: on the conceptual foundations of Shannon's information theory. Synthese 168, 119-149 (2009)

9. Ellerman, D.: The logic of partitions: introduction to the dual of the logic of subsets. Rev. Symb. Log. 3, 287-350 (2010)

10. Ebrahimzadeh, A.: Logical entropy of quantum dynamical systems. Open Phys. 14, 1-5 (2016)

11. Ebrahimzadeh, A.: Quantum conditional logical entropy of dynamical systems. Ital. J. Pure Appl. Math. 36, 879-886 (2016)

12. Ebrahimzadeh, A., Jamalzadeh, J.: Conditional logical entropy of fuzzy $\sigma$-algebras. J. Intell. Fuzzy Syst. 33, 1019-1026 (2017)

13. Good, I.J.: Comment on Patil and Taillie: diversity as a concept and its measurement. J. Am. Stat. Assoc. 77, 561-563 (1982)

14. Patil, G.P., Taillie, C.: Diversity as a concept and its measurement. J. Am. Stat. Assoc. 77, 548-561 (1982)

15. Rao, C.R.: Diversity and dissimilarity coefficients: a unified approach. Theor. Popul. Biol. 21, 24-43 (1982) https://doi.org/10.1016/0040-5809(82)90004-1

16. Tamir, B., Cohen, E.: Logical entropy for quantum states (2014). arXiv:1412.0616v2 [quant-ph]

17. Kolmogorov, A.N.: Foundations of the Theory of Probability. Chelsea, New York (1950)

18. Feller, W.: An Introduction to Probability Theory and Its Applications, vol. II, 2nd edn. Wiley, New York (1971)

19. Walters, P.: An Introduction to Ergodic Theory. Springer, New York (1982)

20. Markechová, D.: The entropy of fuzzy dynamical systems and generators. Fuzzy Sets Syst. 48, 351-363 (1992)

21. Markechová, D.: A note to the Kolmogorov-Sinai entropy of fuzzy dynamical systems. Fuzzy Sets Syst. 64, 87-90 (1994)

22. Markechová, D., Riečan, B.: Entropy of fuzzy partitions and entropy of fuzzy dynamical systems. Entropy 18, Article ID 19 (2016). https://doi.org/10.3390/e18010019

23. Markechová, D., Riečan, B.: Logical entropy of fuzzy dynamical systems. Entropy 18, Article ID 157 (2016). https://doi.org/10.3390/e18040157

24. Markechová, D., Tirpáková, A.: Birkhoff's individual ergodic theorem and maximal ergodic theorem for fuzzy dynamical systems. Adv. Differ. Equ. 2016, Article ID 127 (2016). https://doi.org/10.1186/s13662-016-0855-x

25. Tirpáková, A., Markechová, D.: The fuzzy analogies of some ergodic theorems. Adv. Differ. Equ. 2015, Article ID 171 (2015). https://doi.org/10.1186/s13662-015-0499-2

26. Dumitrescu, D.: Entropy of a fuzzy dynamical system. Fuzzy Sets Syst. 70, 45-57 (1995)

27. Ebrahimi, M.: Generators of probability dynamical systems. Differ. Geom. Dyn. Syst. 8, 90-97 (2006)

28. Ebrahimzadeh, A., Ebrahimi, M.: The entropy of countable dynamical systems. UPB Sci. Bull., Ser. A 76(3), 107-114 (2014) 